\title{
Optimal conditions for imaging in scanning tunneling microscopy: Theory
}

\author{
E. Anguiano, ${ }^{\text {a) }}$ A. I. Oliva, ${ }^{\text {b) }}$ and M. Aguilar ${ }^{\mathrm{c}}$ \\ Instituto de Ciencia de Materiales (CSIC), Campus Universidad Autónoma, 28049 Madrid, Spain
}

(Received 9 April 1998; accepted for publication 18 August 1998)

\begin{abstract}
The scanning tunneling microscopy (STM) feedback system is analyzed to look for the optimal conditions for measurement. The typical feedback circuit normally used in STM and the parameters involved on it are studied, and their relative importance into the loop are discussed. The analysis of the role of each parameter demonstrated the importance of a detailed knowledge of the instrument to assure that the images obtained are reliable. We obtained equations that involve the main parameters of the loop, and yield the optimal conditions for imaging taking into account stability, signal amplitude, and phase shift. Combining the stability conditions with the imaging conditions obtained in this work, we found values for the feedback parameters to perform optimal STM measurements. Moreover, with this work, we highlight the importance to include in further publications the value of the main parameters used to obtain STM images. The conclusion of our work is that in some cases (in particular atomic resolution and fractal analysis) STM users should pay more attention to the setting of the instrument. (C) 1998 American Institute of Physics. [S0034-6748(98)02411-3]
\end{abstract}

\section{INTRODUCTION}

Scanning probe microscopy (SPM) techniques, mainly scanning tunneling microscopy (STM) and atomic force microscopy (AFM), have become today the standard techniques for characterization of surface materials with atomic resolution. Commercial instruments are widely disseminated around the world and their use and importance cover many fields of science. These instruments become increasingly compact and complex. Consequently, the users look to them as "black boxes." In this way, the parameters in the feedback loop are most of the time obtained by trial and error. However, to obtain "real copies" of the surface topography, it is necessary to keep in mind the main characteristics and the limits of the instrument. Pioneering efforts on the STM feedback system analysis were made on the base to find out the stability region ${ }^{1}$ and the transfer function to obtain the conditions for a critical damping ${ }^{2}$ to avoid instabilities. Other works proposed finding out the optimal feedback conditions experimentally-obtaining the critical damping conditions ${ }^{3}$ - or using a mathematical model with complex equations, ${ }^{4}$ without clear conclusions about how to use their results under real conditions. Park and Barret ${ }^{5}$ studied the stability of the tunnel loop by using a resonant model but finally they were not able to acquire the stability region for imaging.

Along this line, we discussed in previous works the optimal conditions in which to have stability, during scanning in the STM, in order to avoid artifacts, instabilities, reso-

\footnotetext{
${ }^{a)}$ Escuela Técnica Superior de Informática, Universidad Autónoma de Madrid, Cantoblanco, 28049, Madrid, Spain; electronic mail: eloy.anguiano@ii.uam.es

b) On postdoctoral fellowship from: CINVESTAV del IPN, Unidad Mérida, AP-73 Cordemex, 97310, Mérida, Yucatán Mexico; electronic mail: oliva@kin.cieamer.conacyt.mx

${ }^{c)}$ Electronic mail: maguilar@icmm.csic.es
}

nance, or noise in the image. ${ }^{6}$ We emphasized the real parameters involved in it and their experimental determination. ${ }^{7}$ Therefore, an extensive analysis of a typical feedback circuit was made, finding conditions and rules for stability during imaging. Because "an image says more than a thousand words," it is important to be sure of the image quality. Thus, a second part was missing in our previous work: the measurement conditions when the feedback loop is under conditions of stability. Therefore, the aim of this work is to present a study on the correct use of the STM feedback system from the point of view of the measurement conditions. As far as we know, this is the first study where the physical parameters used for measurement and the mechanical properties of the STM device have been related between them and with the images obtained.

\section{BACKGROUND}

In this section, the feedback system configuration and the main parameters that affect the measured signal during imaging are explained. In SPM technique, the fine control of the vertical movement of a probe is possible by a feedback loop that needs to be stable and precise during probe scanning. Figure 1 shows a typical block diagram configuration of the feedback control system for a STM as well as the relationships between the parameters in the loop. When the STM is scanning, a small current (tunnel current) is detected when a fine tip (probe) is near to the surface of the material to be analyzed. In the AFM, the tunnel current signal is changed by the mechanical deformation of a small cantilever, which has a tip at the end (probe). In the STM case, the tunnel current is converted to a voltage signal and is amplified by the $I-V$ converter. Then, it is linearized with a logarithmic amplifier (the tunnel current has an exponential behavior with the distance $d$ between probe and sample). The group of parameters is shown in Fig. 1 and it is called factor 


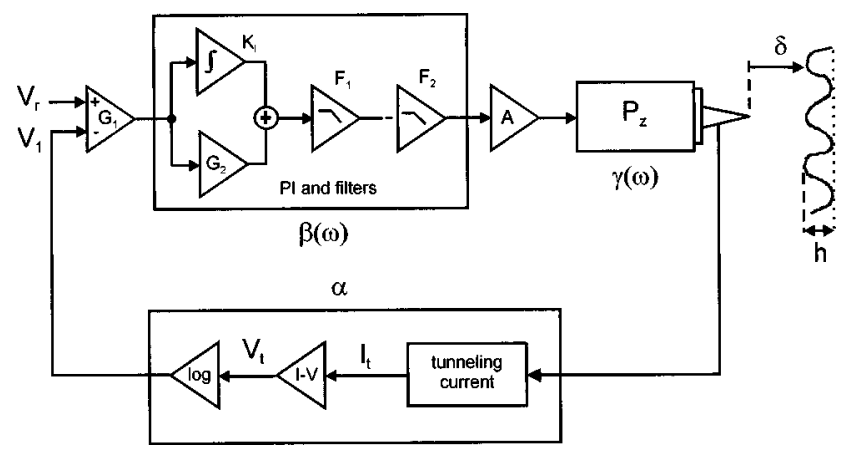

FIG. 1. Typical feedback loop diagram used in a STM.

$\alpha$. The signal is amplified (gain $G_{1}$ ) and summed with a fixed reference voltage $V_{r}$. When scanning, the difference between both signals is a new signal proportional to the error between them. The error signal is generated as a result of the different heights in the sample surface during scanning. The error signal is corrected by the control system in such a way that its value is minimized, i.e., tip-sample distance remains constant. Other elements involved in the feedback loop are the integrator $\mathrm{K}_{1}$ in shunt with the operational amplifier $G_{2}$ and some filters of different order.

The last group of elements is represented in Fig. 1 as a black box with the $\beta(\omega)$ transfer function. The sensitivity of the piezoelectric that controls the tip position is represented by the function $\gamma(\omega)$. It plays an important role in the loop, as we will see later. Sometimes, it is useful to use a high voltage amplifier A to increase the elongation of the piezoelectric, usually in the case of large images. A detailed analysis of the components and stability of the feedback loop for the STM case was published previously elsewhere. ${ }^{8}$

The $\alpha$ and $\beta(\omega)$ factors are defined in terms of known parameters. In the STM case, $\alpha$ is given by the following expression:

$$
\alpha=1.025 K_{L} \log _{10}(e) \sqrt{\phi}=0.445 K_{L} \sqrt{\phi},
$$

where $K_{L}$ is the conversion factor of the logarithmic amplifier (in volts), and $\phi$ the average barrier height (in eV). For our STM system $K_{L}=1 \mathrm{~V}$, and considering the mean value of the barrier height as $4 \mathrm{eV}$ as a typical value then, $\alpha$ $=0.89 \mathrm{~V} / \AA$.

In the AFM case, equation for factor $\alpha$ has another form and depends on the feedback detection based on a laser diode. ${ }^{9}$ This detection system is composed by the laser beam, the lever, the force-sensing tip, the sample, and the photodetector, but it will not be discussed in this work. Similarly, in the STM case, $\beta(\omega)$ is given ${ }^{6}$ by

$$
\beta(\omega)=\left(\frac{K_{1}}{i \omega}-G_{2}\right)\left[\frac{\omega_{c}}{\omega_{c}+i \omega}\right],
$$

where $K_{1}$ is the integration constant, $\omega_{c}$ is the cutoff frequency ( $\mathrm{rad} / \mathrm{s})$ of the low pass filter, and $G_{2}$ is the gain of the amplifier. The integration constant depends on the values of the resistance and the capacitance (this is, the RC factor) used in the electronic circuit and it is given by the relation $K_{1}=1 / R C\left(\right.$ in s $\left.^{-1}\right)$.
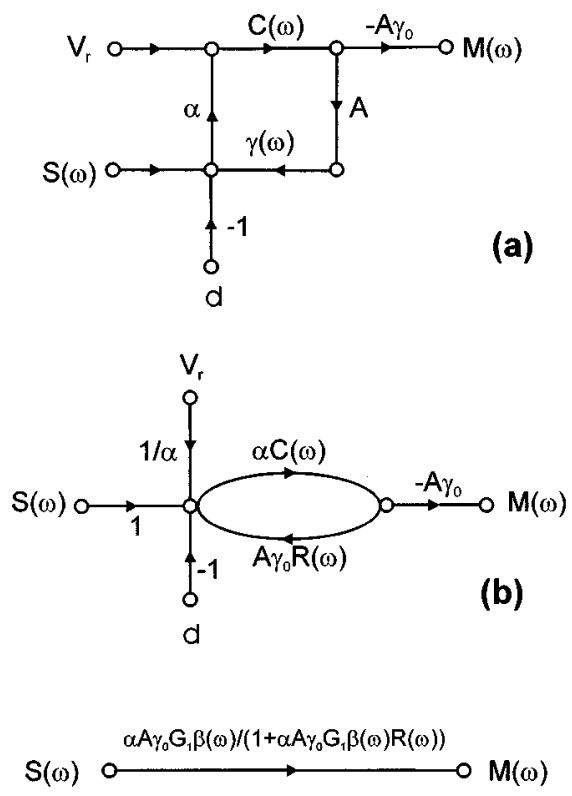

(c)

FIG. 2. (a) Nodal diagram of the feedback loop shown in Fig. 1. (b) Reduction of the nodal diagram presented in Fig. 2(a). (c) New reduction of the nodal diagram presented in Fig. 2(b).

\section{FEEDBACK SYSTEM ANALYSIS}

By using standard control theory, ${ }^{10,11}$ the feedback loop presented in Fig. 1 can be translated into the nodal diagram shown in Fig. 2(a). This will help us to explain, in a simpler way, the following analysis. Note that in Fig. 2(a) new terms have been defined in order to bring together the effect of some parameters. Thus, we defined in the frequency $(\omega)$ space

$$
C(\omega)=-G_{1} \beta(\omega),
$$

where the minus sign represents an inverted signal. Substituting $\beta(\omega)$ by its value given by Eq. (2) then, Eq. (3) converts into

$$
C(\omega)=-G_{1}\left[\frac{K_{1}}{i \omega}-G_{2}\right] .
$$

This value is true if a filter is not used. On the other hand, the piezoelectric element that controls the vertical tip position in the STM case can be modeled by

$$
\gamma(\omega)=\gamma_{0} R(\omega),
$$

where $\mathbf{R}(\omega)$ is the frequency dependent part of the piezoelectric element and $\gamma_{0}$ the voltage sensitivity constant $(\AA / \mathrm{V})$. The piezoelectric behavior, considering two poles for instabilities, can be described as

$$
R(\omega)=\frac{1}{1+i \omega \tau-\left(\omega^{2} / \omega_{0}^{2}\right)},
$$

where, $\omega_{0}$ is the resonance frequency of the piezoelectric. This value is not the resonance frequency of the material, but the first mechanical resonant frequency of the piezoelectric considering the mechanical mounting of the element in the whole STM head. 
If we define $\mathbf{S}(\omega)$ as the real information to be measured on the sample surface (surface topography) and $\mathbf{M}(\omega)$ as the signal measured on the feedback loop then, the ideal case will be that $\mathbf{M}(\omega)$ be the same as $\mathbf{S}(\omega)$. If the optimal conditions of the feedback loop are used, then $\mathbf{M}(\omega)$ will be as close to $\mathbf{S}(\omega)$ as physically possible.

Figure 2(b) shows a reduction to a loop simpler than in Fig. 2(a). To do this, some terms have been grouped. From here on, we can make the approximation that the term $V_{r}$ $-\alpha d \approx 0$ (this means that the error signal is very small). Thus, the loop can be reduced into the simplest loop shown in Fig. 2(c). Now, it can be clearly seen that $\mathbf{S}(\omega)$ and $\mathbf{M}(\omega)$ are linked by the equation

$$
M(\omega)=\left[\frac{\alpha A \gamma_{0} G_{1} \beta(\omega)}{1+\alpha A \gamma_{0} G_{1} \beta(\omega) R(\omega)}\right] S(\omega) .
$$

By defining $G_{0}=\alpha A \gamma_{0} G_{1}$, as the total gain of the closed loop, then Eq. (7) can be rewritten as

$$
M(\omega)=\left[\frac{G_{0} \beta(\omega)}{1+G_{0} \beta(\omega) R(\omega)}\right] S(\omega),
$$

where the term between the parenthesis represents the adimensional transfer function (TF) of the of the feedback system for the case of closed loop and is given by

$$
\operatorname{TF}(\omega)=\frac{G_{0} \beta(\omega)}{1+G_{0} \beta(\omega) R(\omega)} .
$$

We will use this transfer function for further analysis and discussions.

\section{ANALYSIS}

In the analysis of the feedback system, we need to take into account two aspects: (i) to find out the stability region and, (ii) to define limits for the transfer function parameters so that the images measured will be reliable.

\section{A. Stability conditions}

In a previous work, ${ }^{6}$ we analyze the feedback loop to obtain the stability region. An equation to achieve conditions for stability in the feedback loop was obtained and it was the following:

$$
G_{0}\left[\frac{K_{1} \tau+G_{2} \zeta^{2}}{\zeta^{4}+\left(1-\zeta^{2}\right) \omega_{0}^{2} \tau^{2}}\right]<1
$$

where $\zeta$ is the damping factor of the signal caused by the mechanical device and $\tau$ is the delay time or the time necessary for recovery of the signal when an instantaneous perturbation affects the controlled signal. Both parameters can be experimentally obtained and the procedure is explained in detail in Ref. 7.

The value of the damping factor $\zeta$ of the signal is less than 1 for underdamping motion, equal to 1 for critical damping motion, and higher than 1 for over-damping motion. The value is related with the loop parameters by the following relationship: ${ }^{7}$

$$
\left(1-\zeta^{2}\right)=\frac{K_{1}}{K_{1}+G_{2} \tau \omega_{0}^{2}} .
$$

A strong recommendation is to have the damping factor $\zeta$ close to the unit to avoid oscillations in the signal and, consequently, to have the lowest delay time in the response.

From here on, we will consider in the analysis that we know the conditions to work in stable conditions and, then, we use for imaging a sampling frequency $\left(\omega_{s}\right)$ far from and lower than the resonance frequency.

In order to understand our analysis more easily, we will define some adimensional terms as function of the sampling frequency $\omega_{s}$. The new adimensional terms include the elements of the feedback loop. They are defined as

$$
\begin{aligned}
& K=\frac{G_{0} K_{1}}{\omega_{s}}, \quad \tau_{s}=\omega_{s} \tau, \quad W_{0}=\frac{\omega_{0}}{\omega_{s}}, \quad W=\frac{\omega}{\omega_{s}}, \\
& G=G_{0} G_{2} .
\end{aligned}
$$

By using these adimensional parameters, Eq. (11) can be rewritten as

$$
\left(1-\zeta^{2}\right)=\frac{K}{K+G W_{0}^{2} \tau_{s}},
$$

where

$$
\zeta^{2}=\frac{G W_{0}^{2} \tau_{s}}{K+G W_{0}^{2} \tau_{s}} .
$$

By substituting Eqs. (13) and (14) into Eq. (10) for stability and after some algebraic reduction we obtain the same condition, as a function of the new adimensional parameters. The stability condition is now given by

$$
K<W_{0}^{2} \tau_{s}(1-G) .
$$

From this last equation and given that, from its definition, $K$ always needs to be $K>0$, then $G$ necessarily needs to be $G$ $<1$, i.e., $\alpha A \gamma_{0} G_{1} G_{2}<1$.

Equation (15) yields the stability condition. Special cases (for $G_{2}=0$, and for $K_{1}=0$ ) calculated from this equation, give us the same results obtained before in Ref. 7 .

\section{B. Conditions for reliable imaging}

Now we can examine the limits in SPM imaging that are imposed when measurement conditions are taken into account. In fact, the Shannon-Whittaker theorem ${ }^{12}$ establishes the well-defined limits in the measurement of a signal. In the following section, we will discuss these limits.

\section{Amplitude conditions}

The transfer function of the closed loop of the feedback system obtained in Eq. (9) can be written as a function of the adimensional parameters introduced before

$$
T F(W)=-\frac{G+i \frac{K}{W}}{(1-G)-i \frac{K}{W}} .
$$

The squared modulus of the previous complex expression is given by 


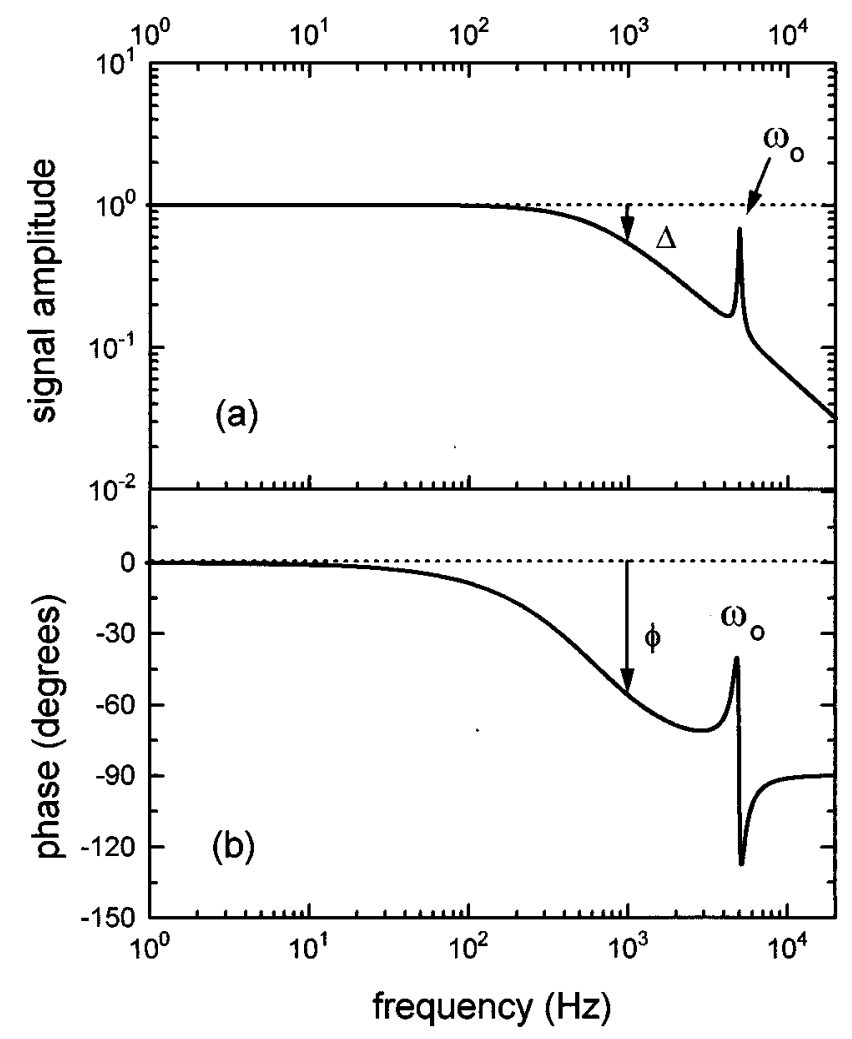

FIG. 3. Typical behavior of the Bode's diagram for the amplitude (a), and phase shift (b) for stable condition.

$$
|T F(W)|^{2}=\frac{G^{2} W^{2}+K^{2}}{(1-G)^{2} W^{2}+K^{2}} .
$$

For a given sampling frequency $\omega_{s}$ at the frequency at which $\omega=\omega_{s}$, by definition, $W=1$. Then,

$$
|T F(1)|^{2}=\frac{G^{2}+K^{2}}{(1-G)^{2}+K^{2}}
$$

Now, we will define a new parameter to be introduced in the analysis called $\Delta$. The new parameter is defined as the variation allowed in the amplitude (attenuation or amplification) with respect to the unitary amplitude on the transfer function at the sampling frequency. Thus the new parameter is given by the equation $|T F(1)|=1+\Delta$. That is, we decide the maximum error in the amplitude to be reached on the transfer function during scanning. Thus, the defined $\Delta$ value can be positive (excess in amplitude) or negative (deficit in amplitude). Note that $T F(W)$ is the amplitude in the Bode's diagram, then for a given $\omega_{s}$, the amplitude in the transfer function can be calculated. The Bode's diagram represents the transfer function of the measured signal by means of the behavior of the amplitude $T F(W)$ and the phase shift $\phi$ as a function of the frequency $\omega$. A typical diagram is shown in Fig. 3 where the Bode's diagram is represented for stable condition. In the amplitude's curve [Fig. 3(a)] for the closedloop condition, the amplitude diminishes as the frequency increases. For a given sampling frequency value, only one value of $\Delta$ can exist. In the particular case of Fig. 3(a) $\Delta$ can only be negative. Therefore, it is important to previously obtain the Bode's diagram in order to know the sign of $\Delta$.
Obviously, the optimal value for this difference in amplitude is $\Delta=0$, that is, $|\mathbf{S}(\omega)|=|\mathbf{M}(\omega)|$, but this is very hard to achieve as we will see later.

A larger difference in the amplitude, with respect to the unitary amplitude, will produce a signal with a larger phase shift [Fig. 3(b)]. At the resonance frequency the amplitude can be higher, and the corresponding phase shift, maximum.

The resonance peaks' position in the Bode's diagram [Fig. 3(a)] gives us information about the election of the sampling frequency to be used. Sampling frequency needs be selected far from and lower than the resonance frequency.

By using the new parameter $\Delta$ in the amplitude at the sampling frequency Eq. (18) can be rewritten by

$$
\frac{K^{2}+G^{2}}{(1-G)^{2}+K^{2}} \leqslant(1+\Delta)^{2} \text { for } \Delta>0
$$

or

$$
\frac{K^{2}+G^{2}}{(1-G)^{2}+K^{2}} \geqslant(1+\Delta)^{2} \quad \text { for } \Delta<0 .
$$

From the last equations, we can write the $K$ value as a function of $\Delta$ and $G$, and then, we obtain two possibilities around the unitary amplitude

$$
K \geqslant \sqrt{\frac{G^{2}-(1+\Delta)^{2}(1-G)^{2}}{(1+\Delta)^{2}-1}} \text { for } \Delta>0
$$

or

$$
K \geqslant \sqrt{\frac{(1+\Delta)^{2}(1-G)^{2}-G^{2}}{(1+\Delta)^{2}-1}} \text { for } \Delta<0 .
$$

Given that, by definition $K$ needs to be real then, the expression inside the square root needs to be positive. This implies that $\Delta$ needs to obey one of the following conditions:

$$
\Delta>\frac{1-2 G}{(1-G)} \quad \text { or } \quad \Delta>\frac{2 G-1}{(1-G)},
$$

depending on the value of $\Delta$ selected (negative or positive, respectively).

The ideal case is when the measured amplitude is equal to the actual amplitude on the surface, i.e., $\Delta=0$ (as it was discussed before). Equation (21) shows that for $\Delta=0$, the value of $G$ will always be $G=1 / 2$ regardless of $K$ value. This is the ideal value of $G=G_{0} G_{2}$ in the feedback loop for a correct SPM imaging. Equation (20) gives the limit of $K$ depending on $\Delta$ and $G$ values.

The Shannon-Whittaker theorem ${ }^{12}$ establishes the maximum sampling frequency in the digitization of a signal. The sampling frequency has to be smaller than two times the value of the cutoff frequency $\omega_{c}$ (frequency at which the amplitude decays $3 \mathrm{~dB}$, i.e., $\Delta=-0.37$ ); otherwise, there will exist an oversampling. Thus, this theorem established an upper limit for $\Delta$ value.

A more detailed discussion about the limits for $\Delta$ and $K$ will be presented in the next section.

\section{The phase conditions}

The phase shift is an important parameter in the feedback loop that should be taken into account. In the design of 
control systems it is usual to provide a phase reserve of about $-45^{\circ}$ at the cutoff frequency, as shown in Fig. 3. This implies that, when $\omega_{s}$ is close to the cutoff frequency, the variation in phase can modify the measured signal. Also, the Shannon-Whittaker theorem ${ }^{12}$ needs to be considered. As mentioned above, this theorem establishes the maximum sampling rate in the digitization of a signal. Because of the relationship between frequency and phase (see Fig. 3), the theorem also imposes an upper limit to the phase shift.

The phase shift $\phi$ between the real information on the sample surface and the measured signal at a given frequency can be calculated from the transfer function obtained previously and shown in Eq. (16). The real and imaginary parts obtained are given by

$$
\operatorname{Re}[\operatorname{FT}(W)]=-\frac{K}{W}\left[\frac{1}{(1-G)^{2}+\frac{K^{2}}{W^{2}}}\right]
$$

and

$$
\operatorname{Im}[\mathrm{FT}(W)]=\left[\frac{K^{2}}{W^{2}}-G(1-G)\right]\left[\frac{1}{(1-G)^{2}+\frac{K^{2}}{W^{2}}}\right] .
$$

Thus, the next relation gives the phase shift between signals

$$
\tan \phi=\frac{K W}{G(1-G) W^{2}-K^{2}} .
$$

At the sampling frequency $(W=1), \phi=\phi_{s}$, the phase shift $\phi_{s}$ is given by

$$
\tan \phi_{s}=\frac{K}{G(1-G)-K^{2}} .
$$

By using Eq. (25), we can obtain the values of $G$ and $K$ for a given sampling phase shift or vice versa. To do this, we must remember the limits imposed to $G$ and $K: 0 \leqslant G<1$, and $K \geqslant 0$. A particular limit for $K$ can be obtained when the phase shift is $\phi=-90^{\circ}$, then, $\tan -90^{\circ}=\infty$. In this case, the solution is simpler than the general one and is given by the relationship

$$
K=\sqrt{G(1-G)} .
$$

The equation represents-in the $(G, K)$ space- a circle centered at $(1 / 2,0)$ and radius $1 / 2$.

\section{RESULTS}

Thus far, we have obtained relations based on real parameters used in the feedback loop to find out the limits for the relevant parameters in order to obtain SPM images with high reliability. Now we will discuss the relationships obtained in the previous section in order to find out the optimal conditions for imaging. In addition, we will discuss some special cases.

\section{A. Optimal conditions}

To have stability in the feedback loop during imaging is as important as obtaining images at the correct sampling frequency. In order to see clearly the role of the equations ob-

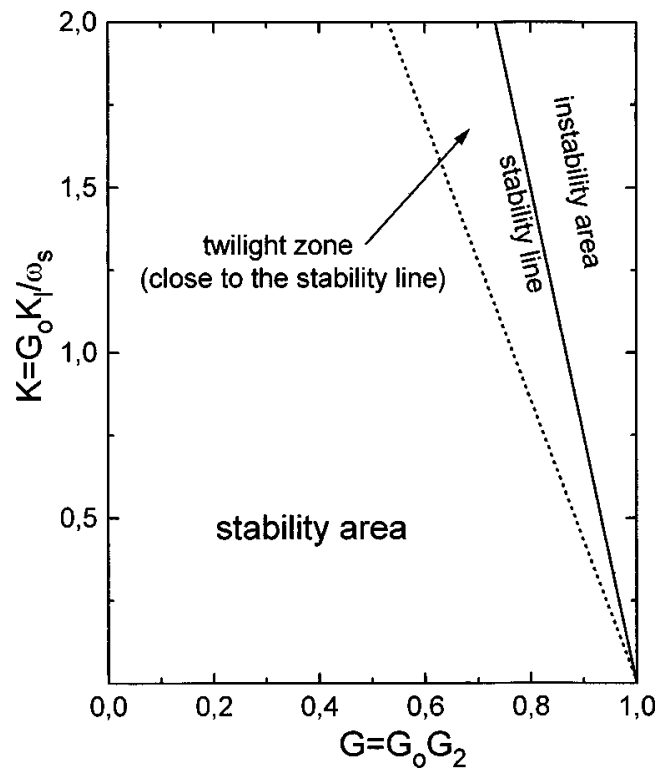

FIG. 4. Limits imposed by the stability condition.

tained for the loop stability, and for the amplitude and phase shift of the signal, we will use particular values for the parameters of the feedback loop. The parameters are in agreement with the Bode's diagram presented in Fig. 3. Here the cutoff frequency appears close to $800 \mathrm{~Hz}$ and the resonant frequency near $5 \mathrm{kHz}$. Then, we will use $1 \mathrm{kHz}$ as sampling frequency $\left(f_{s}\right)$ for our calculations which corresponds to a phase shift of $\phi=-60^{\circ}$, being in agreement with the Shannon-Whittaker theorem. The relations between the parameters $G$ and $K$ are represented for the condition of imaging given by $W_{0}=5, \tau_{s}=0.3, \Delta= \pm 0.5$, and $\phi=-60^{\circ}$. These values imply that $f_{0}=5 \mathrm{kHz}, f_{s}=1 \mathrm{kHz}$, and $\tau$ $=50 \mu \mathrm{s}$.

Naturally, each SPM user needs to use their parameters according to the characteristics of the SPM device used. In addition, the user must fix the desired limits in amplitude and phase shift.

Figure 4 shows the limit for the stability-in the $(G, K)$ space-as a result of the analysis presented before. The area that fulfills the stability conditions given by Eq. (15) is shown. The solid line divides the stability area from the instability area. The slope of the solid line is given by $-W_{0}^{2} \tau_{s}$ and the ordinate at the origin is $W_{0}^{2} \tau_{s}$. Thus, the slope of the solid line will depend on the mechanical resonant frequency of the SPM device, the sampling frequency used, and the time response of the piezoelectric. The area under the solid line represents the region where there is stability in the feedback loop. On the contrary, points above this line give instability conditions in the feedback.

A discontinuous line was drawn close to the solid line. The generated area is an uncertainty area for imaging and will be called the twilight area. Here, the imaging reliability is uncertain because of the equations' validity [remember the approximation made in Eq. (15)]. By working out of the twilight area, it is certain that we avoid instabilities or false information during imaging. Even more, we must select conditions far enough from the stability line.

The limits imposed in the measured signal by the ampli- 


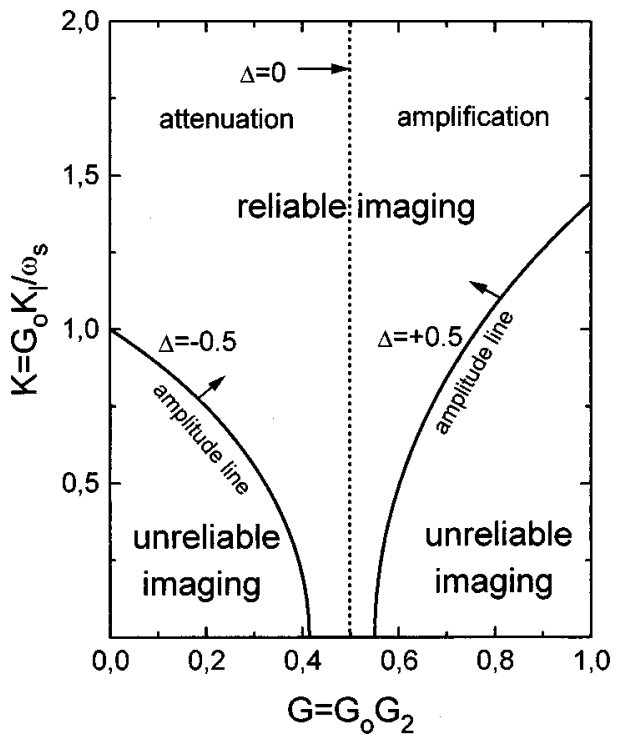

FIG. 5. Limits imposed by the amplitude of the signal. There are two curves corresponding to attenuation and amplification of $\Delta=0.5$.

tude conditions are represented in Fig. 5. The limits represent circular arcs with the center along the $G$ axis [given by Eq. (20)]. The left curve represents the limits in $K$ and $G$ for values in amplitude lower than the selected one for the case of attenuation (i.e., $\Delta<0$ ). The right curve corresponds to the limits for the case of signal amplification (i.e., $\Delta>0$ ). As $\Delta$ value increases, the area for good measurements increases too. Thus, we have more possibilities but the measured signal will be different from the real information, and-quite probably-the Shannon-Whittaker theorem will not be complied with. On the contrary, as the value selected for $\Delta$ decreases, the area for good imaging decreases too. The Shannon-Whittaker theorem establishes that $\omega_{s}<2 \omega_{c}$, however, we recommend a more restricted range: $\omega_{s}<\omega_{c}$. Thus, 0.37 will be the maximum value at the sampling frequency for the amplitude in the attenuation case (i.e., $\Delta=$ -0.37 ) for reliable measurements.

For the special case, in the limit of $\Delta=0$, the circular arcs reach an infinite radius. They have the same value and they are superimposed in the point $G=1 / 2$, and $K=0$; that is, both curves are represented by the vertical broken line drawn at $G=1 / 2$ in Fig. 5. Then, the vertical line divides the areas for the amplitude condition depending on the $\Delta$ value selected (attenuation or amplification). The best conditions for the amplitude will be located along the vertical line.

Figure 6 shows the limits for $K$ and $G$ taking into account the phase shift on the measured signal shown in Eq. (25), for the particular case of $\phi=-60^{\circ}$. In the $(G, K)$ space, Eq. (25) represents a family of circles centered on $[1 / 2$, $\pm 1 /(2 \tan \phi)]$ and radius $[1 /(2 \tan \phi)] \sqrt{\left(\tan ^{2} \phi+1\right)}$.

Similar to the case of the amplitude, for a given maximum phase shift, two areas will exist: the region of $K$ and $G$ values for reliable imaging and the area for unreliable imaging. The reliable region is over the circular arc and the unreliable region is under the circular arc. As the value of the maximum phase shift allowed decreases, the arc gets up to higher $K$ values. This condition implies the use of SPM with higher performance.

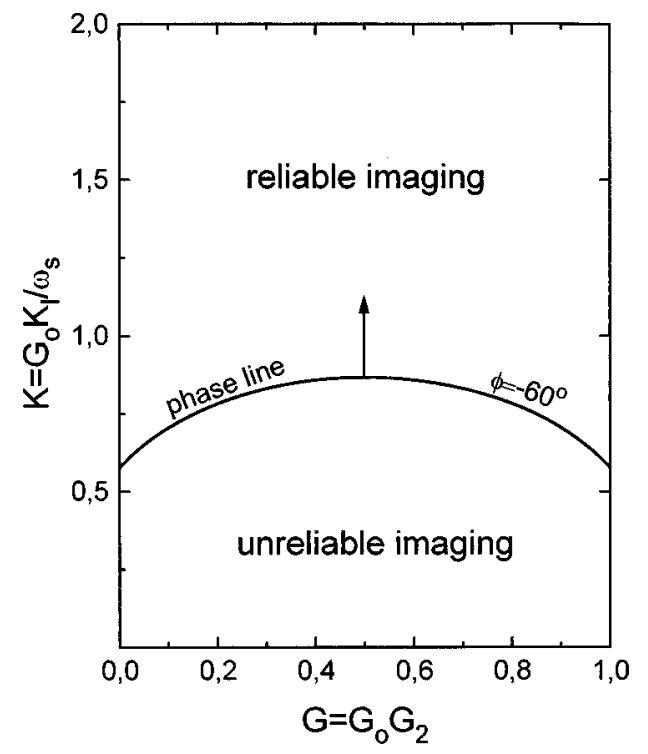

FIG. 6. Limits imposed by the phase shift. The curve corresponds to a maximum phase shift of $-60^{\circ}$.

The case for maximum phase shift of $\phi=-90^{\circ}$ is a circular arc with a maximum at the point $G=1 / 2$ and $K$ $=1 / 2$. This special value on the phase shift always presents a $K=0$ value at the points $G=0$ and $G=1$. High $K$ values for imaging give more possibilities of obtaining images with small phase shift. Again, SPMs with high resonance frequency give more opportunities for good imaging. In all cases, it is recommended that one work far from the stability line to be sure that the equations are valid and to assure that the SPM is working far from the resonance frequency. In order to use high sampling frequencies for scanning, we must use high $K$ values for imaging.

Combining these conditions in the $(G, K)$ space, the intersection of the regions obtained in this work gives an area where the optimal conditions for measurement can be located, depending on the SPM design characteristics. Then, the optimal area is the region in the $(G, K)$ space where the requirements for stability, amplitude, and phase shift are fully complied.

Figure 7 shows the optimal area as a shadowed area. A combination of the main parameters of the feedback loop such that the coordinate $G, K$ fall into the optimal area will give good conditions for loop stability and for imaging. Taking into account that the sampling frequency is included into the definition of the adimensional variables, we know the optimal sampling frequency.

Sometimes it is possible to work out of the optimal area. For example, in the case of the fractal analysis of surfaces, the phase shift is not as important as the amplitude and only the amplitude is critical for a good analysis.

On the contrary, it is possible to work in the region where the amplitude condition is not complied when the $z$ information is not critical. An example: atomic resolution images, where the $x-y$ symmetry is critical to see the surface arrangement and where the distance between atoms needs to be measured.

However, it is very important to know the point where 


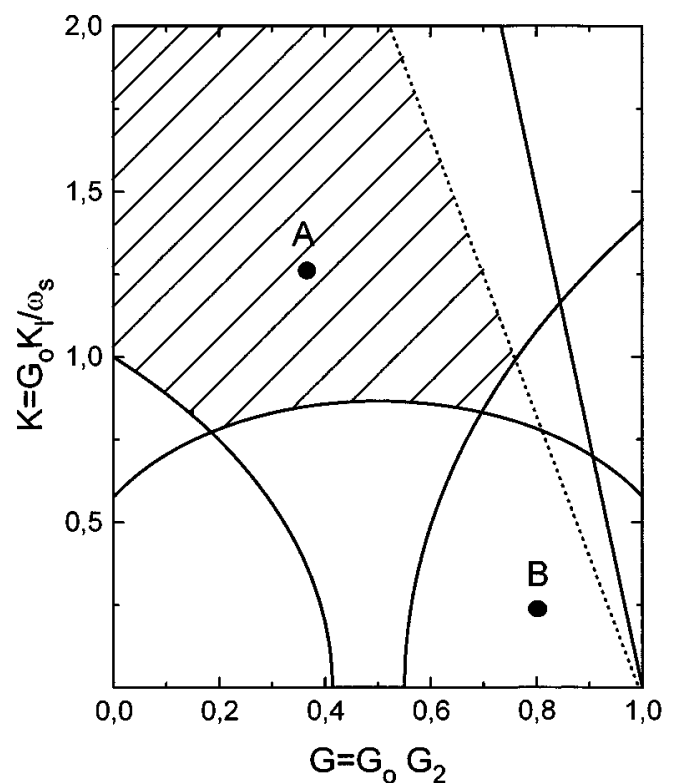

FIG. 7. Optimal area (shadowed area) for imaging obtained by the combination of the limits found for the stability, the amplitude, and the phase shift. Any set of parameters of the feedback loop within this area will give reliable and high quality images.

the SPM is working in order to interpret the results obtained.

The worst region for imaging is the instability area because of the oscillations and noise that can appear in the images.

Figure 7 also considers the possibility of working with only the integrator component in the feedback loop (that is, $G=0$, and working with $K$ values along the vertical axis).

This particular situation forces us to use larger values on $K_{1}$ and $G_{0}$. In this case, it is very important to be sure that the parameters used in the feedback loop are included into the reliable area. This means that the $K$ value needs to be above the intersection of the amplitude line with the $K$ axis. Thus, Fig. 7 gives the optimal region to obtain images within an error in the amplitude lower than \pm 0.5 and a maximum phase shift of $-60^{\circ}$ by using $1 \mathrm{kHz}$ as sampling frequency. In any case, the transfer function (amplitude and phase shift) needs to be found for a given particular condition. Then, the values of the amplitude, the phase shift, and the sampling frequency desirable for imaging can be selected.

As can be seen from these results, it is necessary to know the instrument used for imaging in order to obtain high quality and reliable images.

As an example of the importance of the imaging conditions, two images of the same region of a gold thin film obtained under different conditions are shown in Fig. 8. We use our STM and a control unit developed in our laboratory. The parameters used to obtain them are represented as points $\mathrm{A}$ and $\mathrm{B}$ in Fig. 7. This implies that $K_{1}=999 \mathrm{~s}^{-1}, f_{s}$ $=1 \mathrm{kHz}, \gamma_{0}=300 \AA / \mathrm{V}, G_{1}=0.029, G_{2}=0.05$, and $A=1$. Figure 8(a) was taken with parameters in the region of good conditions (point A, $G=0.4, K=1.25$ ). In this case, the high quality of the image is clearly seen.

On the contrary, Fig. 8(b) corresponds to the same region but it was taken with parameters in the region of bad conditions (point $\mathrm{B}, G=0.8, K=0.25$ ). In this region, neither

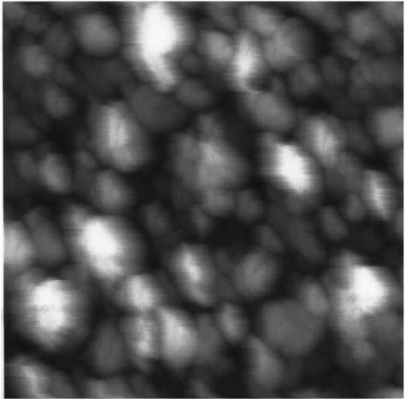

(a)

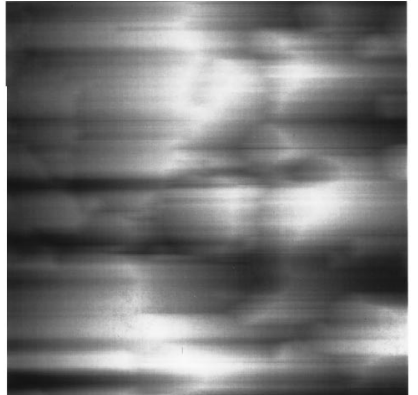

(b)
FIG. 8. (a) STM image of gold thin film obtained with parameters into the reliable area for imaging (point A in Fig. 7). (b) STM image of the same region, but taken with parameters into the bad conditions area (point $\mathrm{B}$ in Fig. 7). Image characteristics: $800 \times 800 \mathrm{~nm}$ size, $256 \times 256$ pixels, $f_{s}$ $=1 \mathrm{kHz}, \gamma_{0}=300 \AA / \mathrm{V}, f_{0}=5 \mathrm{kHz}, \tau=50 \mu \mathrm{s}, A=1$.

the amplitude nor the phase conditions are complied with and it is near the instability line. Note that, in this case, although in conditions with stable loop, the image is very bad and very different from the first one. We used two extremes but a gradual change on the image quality and variations in information have been observed by moving the conditions from site to site in the $(G, K)$ space. A discussion of the errors and implications of imaging in different regions of the $(G, K)$ space in STM, will be the subject of another article. Images in Fig. 8 are only shown to highlight the importance of the measurement conditions.

\section{B. Special cases}

Since the transfer function is given by Eq. (9), if we are very far from the mechanical resonance frequency, i.e., $\omega$ $<\omega_{0}$, then, $R(\omega) \approx 1$. Thus, we have two possible limits that will simplify Eq. (9): (i) $\left|G_{0} \beta(\omega)\right| \ll 1$, and (ii) $G_{0} \beta(\omega) \mid$ $\gg 1$. We will discuss these limits in the following.

\section{Case $\left|G_{0} \beta(\omega)\right| \ll 1$}

In this case $\operatorname{TF}(\omega)=G_{0} \beta(\omega)$, and then

$M(\omega)=\left[G_{0} \beta(\omega)\right] S(\omega)$.

A particular case occurs when the integrator component is much more important than the amplifier $G_{2}$. Thus, if we consider the value of the amplifier as $G_{2}=0$ (i.e., only the integrator), then, in this case Eq. (9) simplifies even more into

$$
M(\omega)=\left[\frac{G_{0} K_{1}}{i \omega}\right] S(\omega) .
$$

The last equation means that the measured signal $\mathbf{M}(\omega)$ is an integrated and out of phase signal proportional to $\mathbf{S}(\omega)$. Therefore, it is possible to obtain high quality STM images with only an integrator. However, the images obtained do not yield the real topography but the integration of it.

This is more important if atomic resolution images are obtained. Typical data used in our SPM for atomic resolution are: $\alpha=0.89 \mathrm{~V} / \AA, A=10, \gamma_{0}=7 \AA / \mathrm{V}, G_{1}=0.1$, and $K_{1}$ $=15 \mathrm{~s}^{-1}$. Under this condition, $G_{0}=\alpha A \gamma_{0} G_{1}=0.62$, and with a typical sampling frequency of $1 \mathrm{kHz}, G_{0} \beta(\omega)$ $\approx 10^{-2}$, i.e., we are in the conditions that produce Eq. (28): 
an integration of the signal. Then, it will be necessary to decrease the sampling frequency below $10 \mathrm{~Hz}$, which means $27 \mathrm{~min}$ are required to obtain a typical image of $128 \times 128$ pixels in order to get out of the region that integrates the signal. Higher scanning speed (or less time for imaging) will give us an integrated and out of phase image as we mentioned before. For this case, $10 \mathrm{~Hz}$ will be a limit for the sampling frequency, below this value $\mathbf{M}(\omega)$ will be the integral of $\mathbf{S}(\omega)$.

\section{Case $\left|G_{0} \beta(\omega)\right| \gg 1$}

Taking into account Eq. (9) and this condition, we obtain that for this special case $\operatorname{TF}\left(\omega_{s}\right) \approx 1$. This implies that $\mathbf{M}(\omega) \approx \mathbf{S}(\omega)$, i.e., we are in the best case.

Since $G_{0}=\alpha A \gamma_{0} G_{1}$, then the condition $\left|G_{0} \beta(\omega)\right| \gg 1$ implies for the case of the sampling frequency that

$$
G_{0} \gg \frac{1}{\left|\beta\left(\omega_{s}\right)\right|} .
$$

That, in terms of the known parameters is

$$
G_{0} \gg \frac{\omega_{s}}{\sqrt{K_{1}^{2}+G_{2}^{2} \omega_{s}^{2}}} .
$$

In addition, Eq. (30) yields the limit for the sampling frequency

$$
\omega_{s} \ll \frac{G_{0} K_{1}}{\sqrt{1-G_{0}^{2} G_{2}^{2}}} .
$$

In other words, values of $\omega_{s}$ that comply with the relationship $\mathbf{M}(\omega)=\mathbf{S}(\omega)$.

From Eq. (31), the maximum sampling frequency for imaging will occur when $G_{0} G_{2}=1$, or when larger values for $G_{0}$ or $K_{1}$ are given. Equation (31) fixes the limit for the sampling frequency $\omega_{s}$ as a function of the feedback control parameters to obtain an image that reproduces exactly the surface topography.

Now, we combine the new limit for $G_{0}$ found for the maximum sampling frequency with the limit for $G_{0}$ to have feedback loop stability. The last limit was previously found and published in Ref. 6 [Eq. (10) in this work]. Then, $G_{0}$ should be between two limits

$$
\left|\frac{\zeta^{4}+\left(1-\zeta^{2}\right) \omega_{0}^{2} \tau^{2}}{K_{I} \tau+G_{2} \zeta^{2}}\right|>G_{0} \gg \frac{\omega_{s}}{\sqrt{K_{I}^{2}+G_{2}^{2} \omega_{s}^{2}}} .
$$

If we do not take into account the limits for sampling frequency when imaging, the result could be an image with distortions.

\section{DISCUSSION}

We obtained relations to find the limits to have good conditions for imaging. By combining these limits, it is possible to achieve an optimal area delimited by the stability, the amplitude, and the phase shift conditions that will permit us to obtain optimal conditions for imaging. For a special case, a relation to find the maximum sampling frequency was determined. From these results, we suggest to include with the SPM images the value of the parameters used to obtain them in order to have more confidence. The image information needs to include at least parameters as sampling frequency, imaged area, and points per line. Some characteristics of the SPM device such as the mechanical resonance frequency $f_{0}$, the piezoelectric sensitivity $\gamma_{0}$, the gain $G_{1}$, the gain $G_{2}$, and the integration constant $K_{1}$ need to be also included. Images published with these conditions will permit us to have more confidence and they will represent a true imaged surface. Similarly, known conditions used in the feedback loop can permit us to distinguish the type of images measured.

\section{ACKNOWLEDGMENTS}

A. I. Oliva thanks CONACyT (México) for the grant given during his postdoctoral fellowship at ICMM-CSIC (Spain). The authors want to thank F. Gómez (Instituto de Ingeniería del Conocimiento) for helpful discussions. This research was supported by the Spanish CICYT, Grant No. MAT96-2514E.

${ }^{1}$ S. Park and C. F. Quate, Rev. Sci. Instrum. 58, 2004 (1987)

${ }^{2}$ Y. Kuk and P. J. Silverman, Rev. Sci. Instrum. 60, 165 (1989).

${ }^{3}$ D. Jeon and R. F. Willis, Rev. Sci. Instrum. 62, 1650 (1991).

${ }^{4}$ A. Hammiche, Y. Wei, I. H. Wilson, and R. P. Webb, Rev. Sci. Instrum. 62, 3010 (1991)

${ }^{5}$ Methods of Experimental Physics: Scanning Tunneling Microscopy, edited by J. A. Stroncio and W. J. Kaiser (Academic, New York, 1993), Vol. 27, Chap. 2.

${ }^{6}$ A. I. Oliva, E. Anguiano, N. Denisenko, and M. Aguilar, Rev. Sci. Instrum. 66, 3196 (1995).

${ }^{7}$ E. Anguiano, A. I. Oliva, M. Aguilar, and J. L. Peña, Rev. Sci. Instrum. 67, 2947 (1996); A. I. Oliva, M. Aguilar, J. L. Peña, and E. Anguiano, Meas. Sci. Technol. 8, 501 (1997).

${ }^{8}$ A. I. Oliva, M. Pancorbo, N. Denisenko, M. Aguilar, V. Rejon, and J. L. Peña, Anales de Fisica 90, 92 (1994).

${ }^{9}$ D. Sarid, Scanning Force Microscopy (Oxford University Press, New York, 1994).

${ }^{10}$ See, e.g., R. C. Dorf, Modern Control Systems, 3rd ed. (Addison-Wesley, Reading, MA, 1983).

${ }^{11}$ K. Ogata, Ingenieria de Control Moderna (Prentice-Hall Hispanoamericana, 1993).

${ }^{12}$ C. E. Shannon, Proc. IRE 37, 10 (1949). 